20 Jan. 1998

IFUP-TH/4-98

hep-ph/9801353

SNS-PH/1998-2

\title{
About the fine-tuning price of LEP
}

\author{
Riccardo Barbieri and Alessandro Strumia \\ Dipartimento di Fisica, Università di Pisa and \\ INFN, sezione di Pisa, I-56126 Pisa, Italia
}

\begin{abstract}
Following Chankowski, Ellis and Pokorski we quantify the amount of fine-tuning of input parameters of the Minimal Supersymmetric Standard Model that is needed to respect the lower limits on sparticle and Higgs masses imposed by negative searches so far, direct or indirect. By including the one loop radiative corrections to the effective potential, the amount of fine-tuning is reduced with respect to the results of CEP by a factor of $2 \div 5$, strongly increasing as $\tan \beta$ approaches 1 . A further reduction factor may come from a more appropriate, less restrictive, definition of the fine-tuning parameter itself.
\end{abstract}

Extensive searches of supersymmetric signals, done mostly, but not only, at LEP, explore in a significant way the parameter space of the Minimal Supersymmetric Standard Model (MSSM). Although variations on the MSSM are certainly possible, the MSSM is, in some of its aspects, representative enough to make these searches relevant in absolute terms, hence the importance of assessing their significance in a quantitative way. In essence, how much should one worry about the fact that no positive result has been found so far? Or, even more importantly, how critical are, to test the MSSM, the searches that will be performed in the nearest future?

A first important attempt to answer these questions has been recently made by Chankowski, Ellis and Pokorski [1] (CEP), who study the minimal 'amount of fine-tuning' [2] $\Delta$ needed to live outside of the MSSM parameter space excluded by the most significant negative searches of supersymmetry done so far. The data taken into account by CEP include:

1. the latest set of precision electroweak tests [3];

2. the latest measurement of the branching ratio B.R. $\left(B \rightarrow X_{s} \gamma\right)$ 《] and, most importantly

3. the lower limits on sparticle and Higgs boson masses from LEP2 [5].

A way to summarise the results of CEP is the following:
- An amount of fine tuning $\Delta$ greater than about 20 is required for any value of $\tan \beta$;

- A scalar Higgs lighter than $90 \mathrm{GeV}$, that will be searched for at LEP in the near future, requires $\Delta \gtrsim 60$

- A value of $\tan \beta$ lower than 2 , a range suggested by an infra-red fixed-point analysis, requires $\Delta$ greater than about 100 .

Since $\Delta$ is supposed to measure, although in a rough way, the inverse probability of an unnatural cancellation to occur in the expression that relates the $Z$ boson mass to the various MSSM parameters, the results of CEP are striking enough to suggest that we take a closer look at them, which is what we do in this letter. This is without underestimating the difficulty of giving an unambiguous quantitative meaning to the 'naturalness bounds' on the sparticle masses, as correctly emphasised by CEP themselves.

Our results are summarised in fig. 1, which gives the prize of the fine tuning $\Delta$ as a scatter plot in the MSSM parameters space still consistent with the data mentioned above and considered by CEP . As seen from fig. 1 , we have a lower limit on $\Delta$ which is about a factor of $5 \div 10$ weaker than that obtained

\footnotetext{
*The density of points in this plot does not have any particular meaning. What counts is rather the enveloping curve at the lower bound of the populated region.
} 


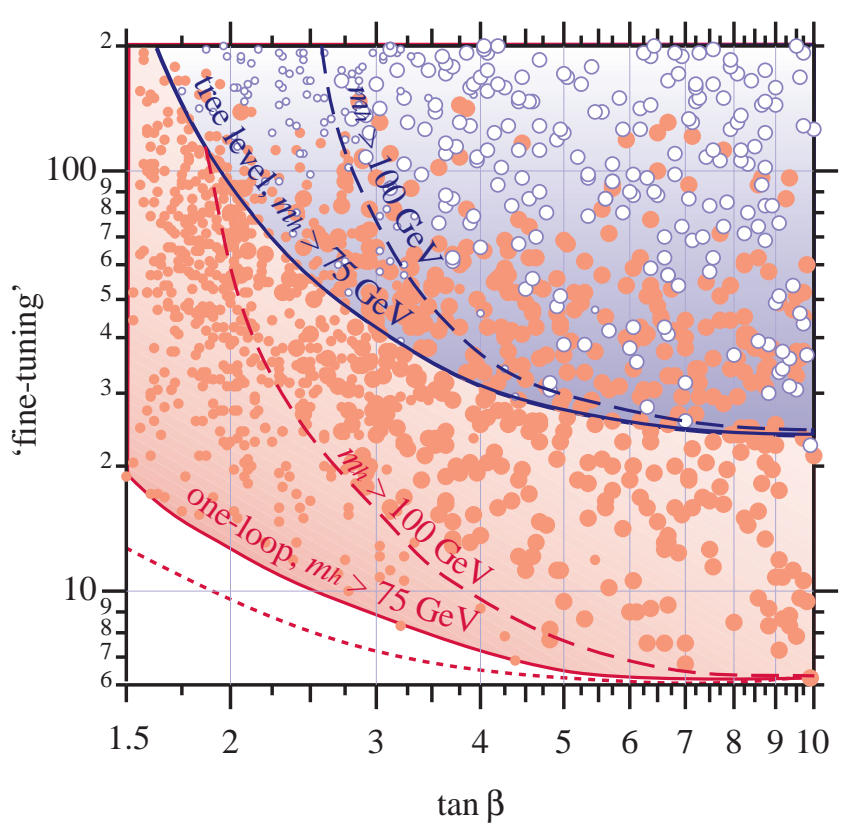

Figure 1: Scatter plot of the fine-tuning as function of $\tan \beta$. In the empty $\circ$ (filled •) points the FT is computed as in CEP (as here). Small points have $m_{h}>75 \mathrm{GeV}$, bigger points have $m_{h}>100 \mathrm{GeV}$. The lower dotted line is explained in the text.

by CEP, a relative factor actually increasing as $\tan \beta$ gets close to 1 . This is mostly due to the inclusion of the full one-loop corrections to the scalar potential. The relative factor between CEP and us also includes an overall factor of about 2 for every $\tan \beta$, coming from what is probably a more adequate definition of the fine tuning parameter itself than the one adopted by CEP (see below).

The inclusion of the full one-loop corrections to the scalar potential [6] is known to be an essential ingredient to make the result not too sensitive to the choice of the renormalization scale of the various low energy parameters [7]. In turn, this has the effect of reducing the fine-tuning parameter especially for values of $\tan \beta$ near to one (where the tree level supersymmetric potential has a flat direction) and for high values of $\Delta$ (because there is a large spread in the superpartner spectrum). Since this is the main source of the difference between CEP and us, this relative reduction effect is shown in fig. 2, also in the form of a scatter plot, for a fixed definition of $\Delta$, no matter which.

The strong increase of the tree-level fine-tuning at small values of $\tan \beta$ is not due to the fact that the experimental constraints are stronger at small $\tan \beta$, but to the particular nature of the tree level MSSM potential: its super-gauge part vanishes at $\tan \beta=1$. For this reason, in tree-level approxima-

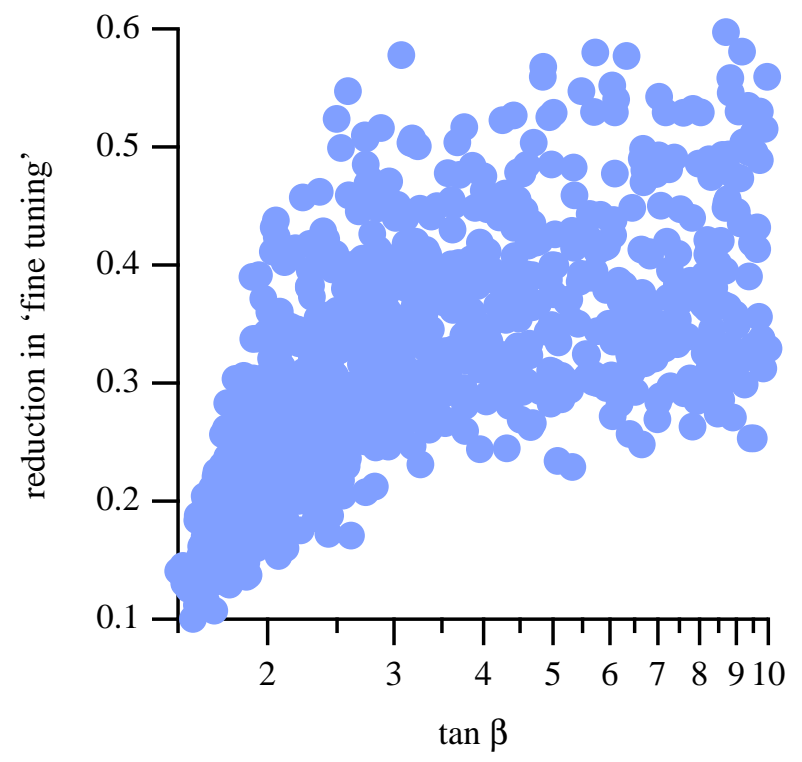

Figure 2: Reduction in fine-tuning due to one-loop effects. The parameters of the tree level potential have been renormalized at $Q=175 \mathrm{GeV}$.

tion the second derivative of the potential is small at $\tan \beta \sim 1, V^{\prime \prime} \sim M_{Z}^{2} \cos ^{2} 2 \beta \sim m_{h}^{2}$, and a variation of the MSSM parameters induces a very large variation in $M_{Z}^{2}$, dominantly through the induced variation of $\tan \beta$. To illustrate this point, we have also computed the logarithmic derivative that defines the fine tuning at fixed values of $\tan \beta$. The minimal experimentally allowed value of this biased fine tuning, represented by the dotted line in fig. 1 , does not strongly increase at small $\tan \beta$, and is comparable to the one-loop unbiased value (continuous line). The reduction in fine-tuning due to one-loop effects is larger if the sign of $\mu$ is positive and is roughly given by $\Delta_{\text {loop }} / \Delta_{\text {tree }}=m_{h \text { tree }}^{2} / m_{h \text { loop }}^{2}$.

The definition of $\Delta$ in terms of the logarithmic sensitivities of $M_{Z}$ with respect to variations of a set of input parameters $\{\wp\}$ ( $\mu$-term, soft terms, gauge and Yukawa couplings) chosen to be the "fundamental" ones has been criticized [9] as being too restrictive or unadequate at all. Different definitions give bounds weaker by a factor that can go up to $3 \div 4$. Partly, this is where the ambiguity of the quantitative concept comes in. We stick to a definition 10 that avoids these criticisms by replacing the logarith-

\footnotetext{
†This different choice of the basic 'input' parameters can also be a correct one. For example the pseudo-Goldstone mechanism for a light Higgs doublet links the tree level parameters of the Higgs potential so that $\tan \beta=1$.
} 


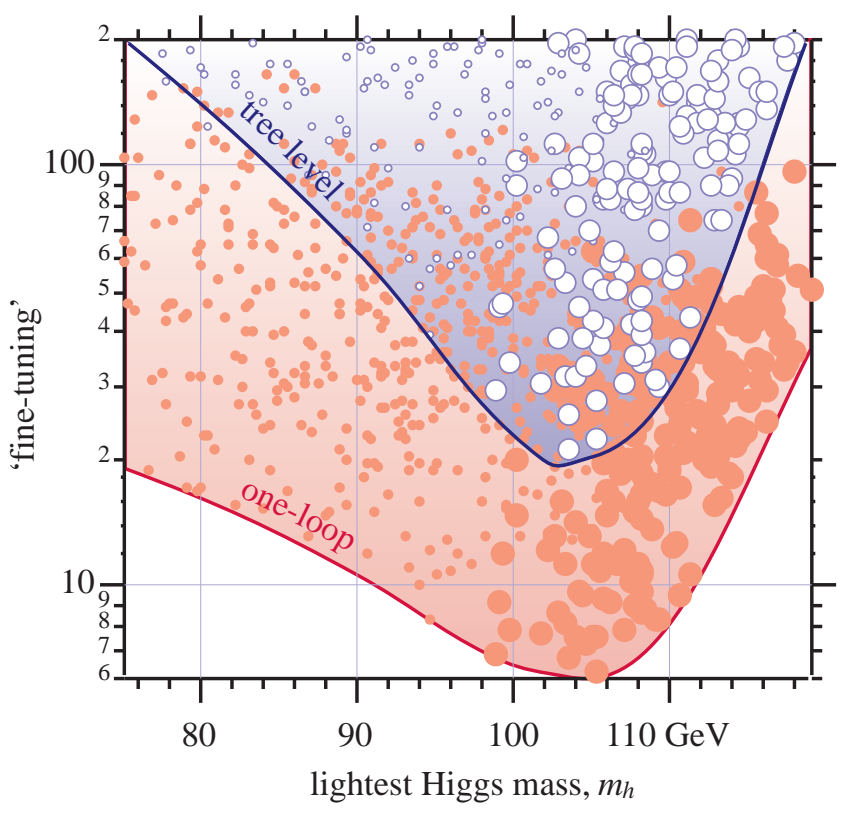

Figure 3: Scatter plot of the fine-tuning as function of $m_{h}$. In the empty $\circ$ (filled $\bullet$ ) points the FT is computed as in CEP (as here). Small points have $\tan \beta<4$, bigger points have $\tan \beta>4$.

mic derivatives present in the original definition

$$
d=\left|\frac{\mu}{M_{Z}^{2}} \frac{d M_{Z}^{2}}{d \mu}\right|
$$

with a particular finite difference

$$
\Delta=\left|\frac{\mu^{2}}{M_{Z}^{2}} \frac{\Delta M_{Z}^{2}}{\Delta \mu^{2}}\right|
$$

explicitly defined in [10]. In practice $2 \Delta$ reduces to $d$ when $\Delta \gg 1$ and is up to $30 \%$ lower than it in all cases of interest. This definition more directly weights as unnatural the possibility of cancellations between different contributions to the expression of the $Z$-boson mass. To make a long story short, we say that there is an unnaturally small probability $p \approx \Delta^{-1}$ that every single 'contribution' to $M_{Z}^{2}$ be $\Delta$ time bigger than their sum, $M_{Z}^{2}$. This explains a factor of 2 between us and CEP. The fine-tuning with respect to the ' $\mu$-term' parameter is the one that gives the strongest constraint.

As pointed out by CEP, a key search to be performed in the nearest future is the one of the lightest MSSM Higgs boson at LEP2. The impact of this search is illustrated in fig. 3 for every value of $\tan \beta$ less than 10. The analysis for large values of $\tan \beta$ is beyond the scope of this letter. The low $\Delta$-values for $m_{h} \gtrsim 90 \mathrm{GeV}$ in fig. 3 correspond to moderately large values of $\tan \beta$ (but still below 10). To explore the $h$-mass region up to $100 \mathrm{GeV}$ is known to be a very significant study of the low $\tan \beta$ region. This is illustrated by comparing the dashed lines with the continuous ones in fig. 1, where the only difference is the constraint that $m_{h}>100 \mathrm{GeV}$ (dashed lines) rather than the present limit, $m_{h}>75 \mathrm{GeV}$ (continuous lines). The values of $m_{h}$ have been computed using the approximation presented in [11].

As stressed in [1] the fine-tuning bounds are not much different in non minimal supergravity models 12. In gauge-mediation models the bound on the right-handed slepton masses becomes the relatively most relevant constraint. Its main effect is that, in minimal gauge mediation, $\Delta<10$ can only be achieved if the messenger scale $M_{M}$ is sufficiently high, $M_{M} \gtrsim 10^{12} \mathrm{GeV}$, or extremely low [10].

In conclusion we confirm the significance of the negative searches performed so far to look for a supersymmetric particle spectrum in a direct or an indirect way. In relative terms, adopting a quantitative measure of the naturalness criterium to assess their significance, the searches for charginos and neutralinos at LEP2 have played the most significant role so far. Nevertheless, regions of the MSSM parameter space with a relatively high "naturalness probability" still exist. The need to explore them confirms the importance of looking at LEP2 for the highest possible Higgs masses.

\section{References}

[1] PH. Chankowski, J. Ellis and S. Pokorski, hepph/9712234

[2] J. Ellis, K. Enqvist, D.V. Nanopoulos and F. Zwirner, Nucl. Phys. B276 (1986) 14; R. Barbieri and G.F. Giudice, Nucl. Phys. B306 (1988) 63.

[3] D. Ward, plenary talk at the Europhysics Conference on High-Energy Physics, Jerusalem, aug. 1997.

[4] M.S. Alam et al., Phys. Rev. Lett. 74 (1995) 2885.

[5] P. Janot, plenary talk at the Europhysics Conference on High-Energy Physics, Jerusalem, aug. 1997; open session of the LEP experiments committee, nov. 1997.

[6] R. Arnowitt and P. Nath, Phys. Rev. D46 (1992) 3981; V. Barger, M.S. Berger and P. Ohmann, Phys. Rev. D49 (1994) 4908.

[7] G. Gamberini, G. Ridolfi and F. Zwirner, Nucl. Phys. B331 (1990) 331.

[8] K. Inoue, A. Kakuto and T. Takano, Progr. Theor. Phys. 75 (1986) 664; A. Anselm and A. Johanson, Phys. Lett. B200 (1988) 664.

[9] B. de Carlos and J.A. Casas, Phys. Lett. B309 (1993) 320; G.W. Anderson and D.J. Castaño, Phys. Lett. B347 (1995) 300.

[10] P. Ciafaloni and A. Strumia, Nucl. Phys. B494 (1997) 41.

[11] M. Carena, J.R. Espinosa, M. Quiros, C.E.M. Wagner, Phys. Lett. B355 (1995) 209.

[12] S. Dimopoulos and G. Giudice, Phys. Lett. B357 (1995) 573. 